\title{
Guava Leaf Extracts Inhibit 3T3-L1 Adipocyte Differentiation Via Activating AMPK
}

\author{
Hisae Yoshitomi ${ }^{\mathrm{a}}$, Lingling Qin ${ }^{\mathrm{b}}$, Tonghua Liu ${ }^{\mathrm{b}}$ and Ming Gao ${ }^{\mathrm{a},{ }^{*}}$ \\ ${ }^{a}$ School of Pharmaceutical Sciences, Mukogawa Women's University, 11-68 Koshien Kyuban-cho, \\ Nishinomiya, Hyogo 663-8179, Japan \\ ${ }^{b}$ Beijing University of Chinese Medicine subsidiary Dongfang Hospital, Beijing, China
}

\begin{abstract}
The guava tree (psidium guajava linn.) is commonly used not only as food but also as folk medicine. In our previous studies, we showed that oral administration of guava leaf extracts (GLE) had beneficial anti-obesity effects using metabolic syndrome model rats. However, we did not clarify molecular mechanism by which GLE administration leads to anti-obesity effect. This study was designed to evaluate the mechanism of anti-obesity by GLE using 3T3-L1 pre-adipocyte cell lines. We found that GLE significantly inhibited 3T3-L1 differentiation via down-regulation of adipogenic transcription factors and markers. Mitotic clonal expansion, which is essential for adipose differentiation, was also depressed in the early phase. Interestingly, GLE increased the phosphorylation of AMPK on 3T3-L1 cells and, by pretreatment with AMPK siRNA, the GLE treatment group showed restored adipocyte differentiation. In conclusion, these results showed that GLE is capable of inhibiting adipocyte differentiation via AMPK activation and therefore it may prevent obesity in vivo.
\end{abstract}

Keywords: AMP-activated protein kinase, 3T3-L1 differentiation, guava leaf extract, Mitotic clonal expansion, obesity.

\section{INTRODUCTION}

The guava tree (psidium guajava linn.) is a member of the Myrtaceae family, which ranges over tropical and subtropical countries. It is commonly used not only as food but also as folk medicine with antioxidant, antispasmodic, anti-allergy, anti-inflammatory and antidiabetic properties using extracts from the fruit, leaf, bark or roots [1, 2]. Leaves of this plant have been used as folk medicine and are reported to contain several compounds such as various terpenoids [3, 4], flavonoids [5] and tannins [6]. In our previous studies, we showed that oral administration of guava leaf extract (GLE) had beneficial anti-obesity effects, preventing body weight gain, reducing adipose tissue weight and fatty liver, and also improving insulin resistance using SHRSP.Z-Leprfa/lzmDmcr rats, which were established as a new model of metabolic syndrome [7]. We did not, however, clarify how to improve obesity by GLE administration.

As one of the mechanisms of anti-obesity, reducing fat accumulation through the inhibition of adipocyte differentiation may play a crucial role. Several previous studies have established preadipocyte cell lines such as 3T3-L1, increasing our understanding of adipocyte differentiation. Peroxisome proliferator-activated receptor gamma (PPARy), from the CCAAT-enhancer-

${ }^{*}$ Address corresponding to this author at the School of Pharmaceutical Sciences, Mukogawa Women's University, 11-68 Koshien Kyuban-cho, Nishinomiya, Hyogo 663-8179, Japan; Tel/Fax: +81 79845 9983;

E-mail: gaoming@mukogawa-u.ac.jp binding protein (C/EBP) family, was reported as a transcription regulator of adipocyte differentiation. In particular, it was revealed that PPARy is a transcription factor that is indispensable for the differentiation of fat cells, and it is considered that C/EBPa is a transcription factor with an essential role in the development of insulin sensitivity. Of these factors, adipocyte specific fatty acid binding protein (aP2) is a fatty acid binding protein that functions in fatty acid import, storage and export [8, 9]. Growth-arrested preadipocytes reenter the cell cycle and undergo division, known as mitotic clonal expansion, which is necessary for adipogenesis [10]. These are also used as adipogenic markers to detect mature adipocytes.

AMP-activated protein kinase (AMPK) plays a key role in the regulation of energy homeostasis [11]. AMPK activation requires phosphorylation at Thr172 of AMPKa subunit and it acts on metabolic enzymes and transcription factors [12]. Acetyl-CoA carboxylase (ACC), which is a critical enzyme for lipid biosynthesis, can be phosphorylated and inactivated by AMPK [13]. Fatty acid oxidation was increased by inhibition of carnitin palmitoyl-CoA transferase (CPT1) via inactivation of ACC [14]. Previous studies have demonstrated that AMPK activation by some compounds were associated with adipocyte differentiation.

In the present study, we used 3T3-L1 cell to determine the effects of GLE on anti-obesity by adipocyte differentiation and changes in the expression 
of PPARy, C/EBPa and aP2 genes. Also, AMPK siRNA was applied to identify the function of AMPK in the regulation of adipocyte differentiation by GLE.

\section{MATERIALS AND METHODS}

\section{Cell Line}

3T3-L1 preadipocytes were purchased from The Japanese Collection of Research Bioresources Cell Bank (Osaka, Japan).

\section{Reagents}

Guava leaf $70 \%$ ethanol extracts were supplied by Beijing University of Chinese Medicine, which identified the biological materials. Dulbecco's modified Eagle's medium (DMEM) and antibiotic solution were obtained from Nacalai Tesque (Kyoto, Japan). Insulin was also obtained from Nacalai Tesque. Isobutylmethylxanthine (IBMX) and dexamethasone (DEX) were purchased from Wako Pure Chemicals (Osaka Japan). Antibodies against AMPKa-Thr172, AMPKa, ACC-Ser79, and ACC were purchased from Cell Signaling Technology (Beverly, MA, USA). Real-time PCR kits were from TOYOBO (Tokyo, Japan) and primers were provided by Invitrogen (CA, USA).

\section{Cell Culture and Adipocyte Differentiation}

The 3T3-L1 cells were cultured in DMEM containing $10 \%$ FBS under $37{ }^{\circ} \mathrm{C}, 5 \% \mathrm{CO}_{2}$ and $95 \%$ humidity. Two days after cells reached confluence, adipocyte differentiation was induced with insulin, DEX and IBMX in DMEM. After 2 days, medium was replaced with DMEM containing only insulin, and after a further 2 days, the medium changed with regular medium. 3T3 cells were treated with different concentrations of GLE $(10,50,100 \mu \mathrm{g} / \mathrm{mL})$ during the differentiation process.

\section{Oil Red O Staining of 3T3-L1 Cells}

Lipid accumulation in differentiated adipocytes can be assessed by staining neutral fats using the dye Oil red $O$. Adipocytes were fixed in $10 \%$ formalin and then stained for 20 min with dye. Lipid staining was quantified by adding isopropanol to extract the dye and measuring absorbance at $510 \mathrm{~nm}$.

\section{Primary Antibodies Used}

Immunoblotting was performed with the following commercially available antibodies: anti-rabbit AMPK, anti-rabbit phosphor-AMPK, anti-rabbit ACC, anti-rabbit phosphor-ACC from Cell Signaling Technology. Antimouse $\beta$-actin was obtained from Sigma (St. Louis, MO, USA).

\section{Western Blot Analysis}

The cells were homogenized with ice-cold homogenized buffer containing $50 \mathrm{mM}$ Tris- $\mathrm{HCl}(\mathrm{pH} 7.4)$, $100 \mathrm{mM} \quad \mathrm{NaCl}, \quad 1 \%$ Nonidet-P40, $0.25 \% \quad \mathrm{Na}$ deoxycholate, $0.1 \%$ SDS, $1 \mathrm{mM}$ EDTA, $50 \mathrm{mM} \mathrm{NaF}$, $2 \mathrm{mM} \mathrm{Na}_{3} \mathrm{VO}_{4}, 30 \mathrm{mM}$ Na pyrophosphate, 2mM PMSF, $1 \mathrm{mM}$ benzamidine, $\quad 0.02 \mathrm{~g} / \mathrm{mL}$ trypsin inhibitor, $0.02 \mathrm{~g} / \mathrm{mL}$ leupeptin, and $0.02 \mathrm{~g} / \mathrm{mL}$ aprotinin. After incubation for 2 hours, lysates were centrifuged at $15,000 \mathrm{rpm}$ for $20 \mathrm{~min}$ and supernatants were isolated. Proteins were extracted by boiling the tissues in $0.5 \mathrm{mmol} / \mathrm{l}$ Tris $/ \mathrm{HCl}$, pH6.8, glycerol, $10 \%$ SDS, $0.1 \%$ bromophenol blue and 2-mercaptethanol. The proteins $(25 \mu \mathrm{g} / \mathrm{lean})$ were electrophoresed using $7.5 \%-12.5 \%$ SDS-PAGE gel at $100 \mathrm{~V}$ for 2 hours. After fractionating, the proteins were transferred onto a PVDF membrane (Amersham Life Science Inc.) at 100mA for 2 hours. The membrane was blocked in Blocking One (Nacalai Tesque) for $20 \mathrm{~min}$. After appropriate blocking, the blot was incubated with the primary antibody in antibody solution 1 (Toyobo) overnight. It was then washed with TTBS and finally incubated for $1 \mathrm{~h}$ with a 1:5000 dilution of anti-rabbit and mouse IgG-horseradish peroxidase. Detection was performed using an ECL kit (Amersham Inc.). $\beta$-actin was used as an internal control. The density of the bands was measured using NIH Image.

\section{Real-Time RT PCR}

Total RNA was extracted from cells using SepasolRNA I Super G (Nacalai Tesque). From each sample,

Table 1: Primer Sequences

\begin{tabular}{|c|c|c|}
\hline Gene & Forward & Reverse \\
\hline \hline PPAR-y & CCAACTTCGGAATCAGCTCTGT & AGGCTCTTCATGTGGCCTGTT \\
\hline C/EBP- $\alpha$ & CGCCTTCAACGACGAGTTC & TTGGCCTTCTCCTGCTGTC \\
\hline aP2 & AGCATCATAACCCTAGATGGCG & CATAACACATTCCACCACCAGC \\
\hline 36B4 & AGCCTTCCTTCTTGGGTATG & CAGGAGGAGCAATGATCTTG \\
\hline
\end{tabular}


total RNA was reverse transcribed to cDNA using a ReverTra Ace qPCR RT Kit (Toyobo). THUNDERBIRD SYBR qPCR Mix was used for quantitative real-time RT-PCR analysis of each gene expression. The primers are listed in Table 1. Amplification was performed with a real-time PCR system (ABI Prism 7000). Results are expressed as a relative value after normalization to 36B4 expression.

\section{AMPKa siRNA Transfection}

Preadipocytes were seeded onto the plate and siRNA transfection was carried out 2 days after the confluence of preadipocytes. Transfection reagents and siRNA were individually diluted and mixed. Mixtures were added to each well and incubated for 6 hours. Normal growth medium was added, incubated

\section{A}

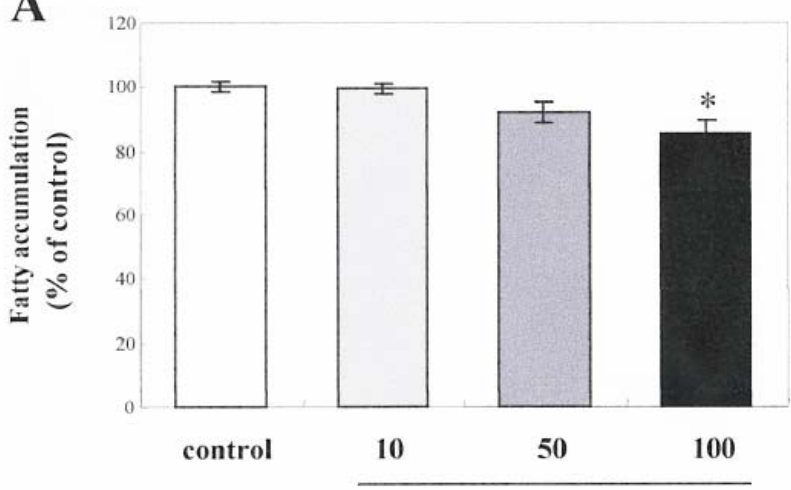

GLE (ug/mL) for 24 hours, and media were removed and replaced with induction medium or GLE.

\section{Statistical Analysis}

Data are expressed as the mean \pm SEM. Statistical analysis of the data was performed by Student's $t$-test for the difference between two groups or Dunnett's test for multiple comparisons. A $p$-value less than 0.05 was considered significant.

\section{RESULTS}

\section{GLE Inhibited 3T3-L1 Differentiation}

The effects of GLE on adipocyte differentiation were examined by Oil red $O$ staining. The results showed that GLE inhibited the accumulation of lipid droplets in

\section{B}

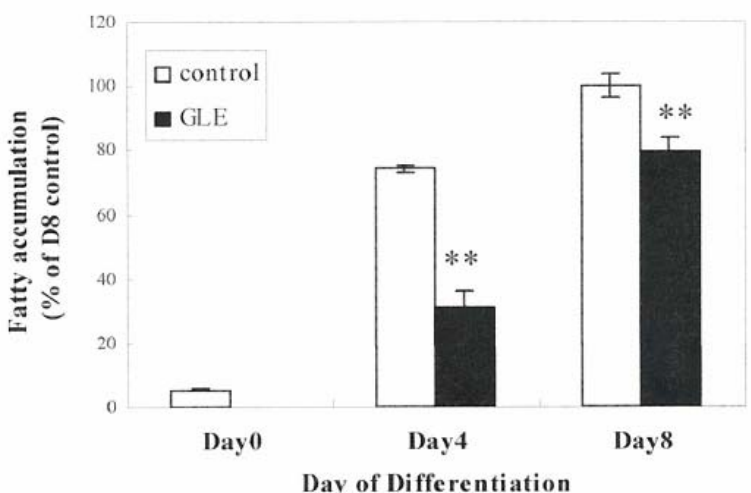

C

Day 0

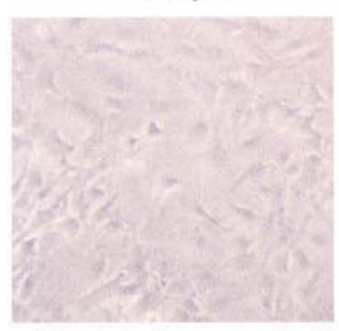

Day 4

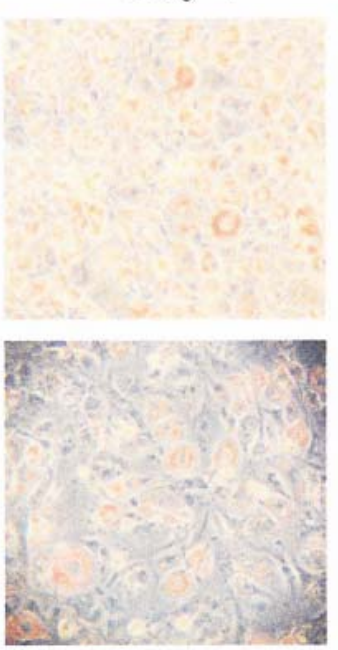

Day 8

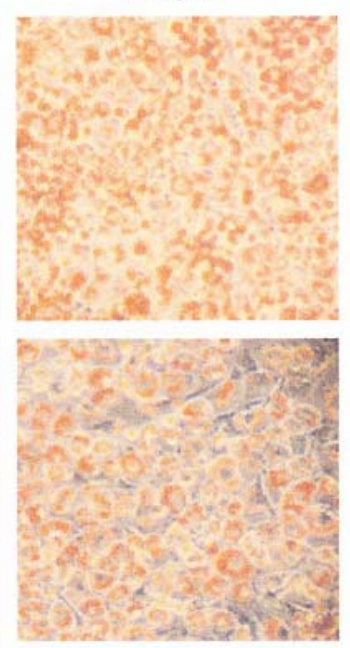

control

\section{GLE}

Figure 1: Effect of GLE on adipocyte differentiation in 3T3-L1 cells.

Confluent cells were exposed to a differentiation cocktail and GLE at the indicated concentrations $(\mathbf{A})$ or cells were exposed to GLE $(100 \mathrm{ug} / \mathrm{mL})$ for the indicated time period $(\mathbf{B})$. Fat drops were stained by Oil red $\mathrm{O}$ dye staining (C). Data are the means \pm SEM * $P<0.05^{* *} P<0.01$ vs control group; $\mathrm{n}=3$. 
A. PPAR gamma

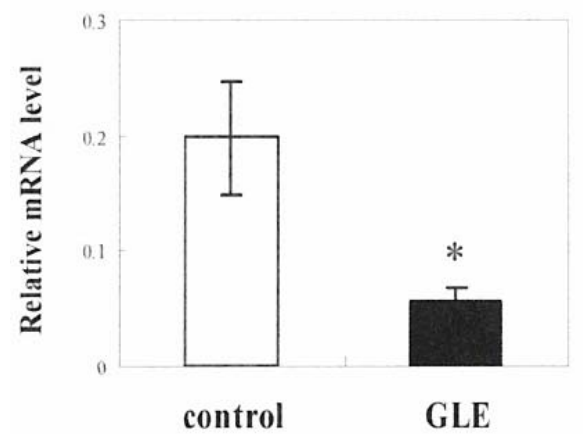

C. aP2

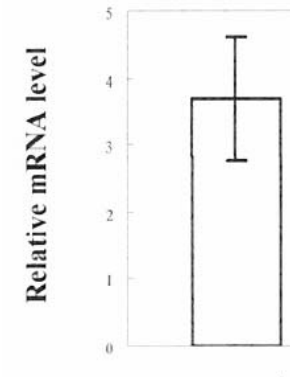

control

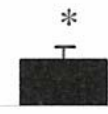

GLE
B. C/EBP alpha

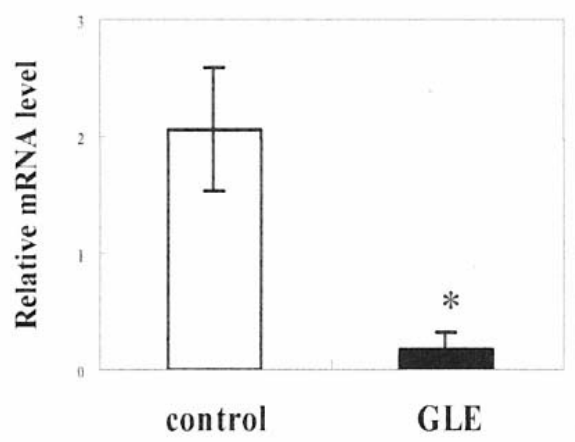

D. ACC

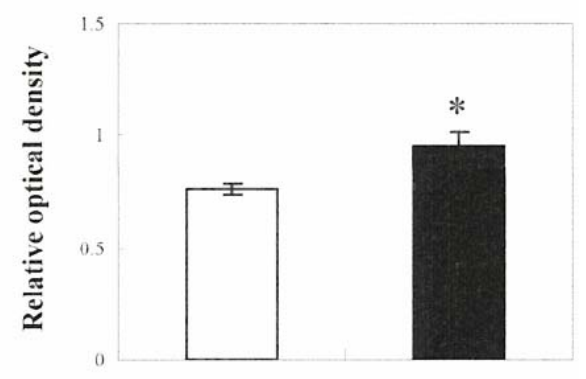

control

GLE

Figure 2: GLE Inhibited the Expression of Adipogenesis Related Transcription Factors and Markers.

3T3-L1 cells were induced to differentiate and were treated with or without GLE (100 ug/mL). Total RNA and protein were extracted on day 4 and the effects on mRNA levels of PPAR-gamma (A), C/EBP-alpha (B) and aP2 (C) were investigated using real-time PCR. The protein level of ACC and its phosphorylated state (D) were determined by Western blotting. Data are the means $\pm \mathrm{SEM} ;{ }^{*} P<0.05$ vs control group; $\mathrm{n}=3$.

a dose-dependent manner during 3T3-L1 differentiation (Figure 1A). On Day 4, the addition of GLE cells decreased intracellular fatty accumulation as compared with the control (Figure 1B). This result was further supported by photographs of lipid staining (Figure 1C).

\section{GLE Inhibited the Expression of Adipogenesis- Related Transcription Factors and Markers}

3T3-L1 adipogenic differentiation requires the synergistic action of multiple transfection factors and adipogenic markers including PPARY, C/EBPa and

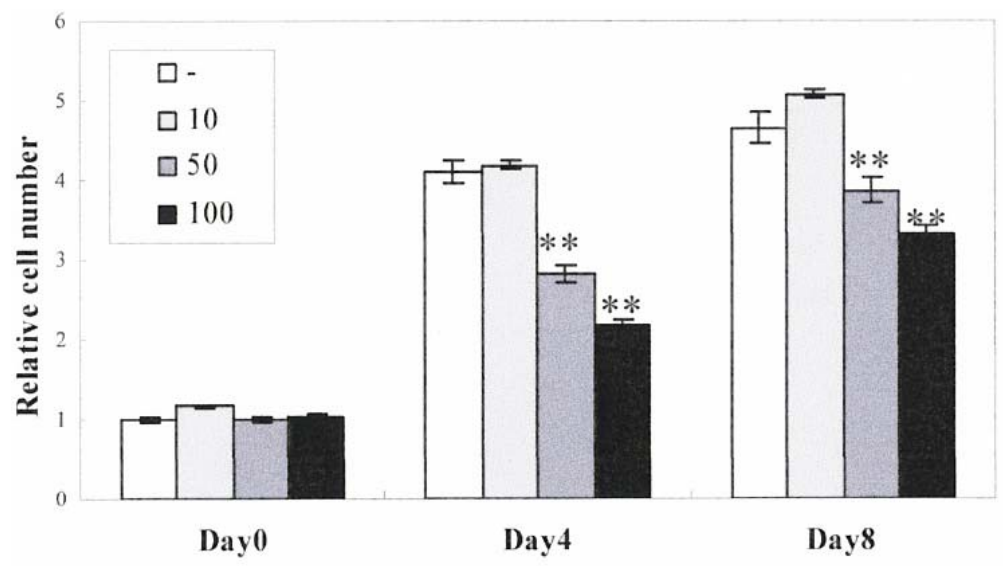

Day of Differentiation

Figure 3: Effect of GLE on clonal expansion in 3T3-L1 cells.

Cells were differentiated at the indicated GLE concentration $(10,50,100 \mathrm{ug} / \mathrm{mL})$. On Day 0 , 4, 8, cell numbers were counted using the MTT assay. Data are the means \pm SEM; ${ }^{* *} P<0.01$ vs control group; $\mathrm{n}=3$. 
aP2. Cells incubated with $100 \mu \mathrm{g} / \mathrm{mL}$ GLE exhibited markedly decreased mRNA expression of PPARY, $\mathrm{C} / \mathrm{EBPa}$ and aP2 compared with the control, as demonstrated by real-time PCR (Figure 2A, B, C). GLE treatment also resulted in a significant increase in the phosphorylation of ACC, which is a late adipogenic marker enzyme in 3T3-L1 (Figure 2D).

\section{GLE Inhibited Clonal Expansion}

In order to examine whether the site of action of GLE is within this initiation phase of adipogenesis, cell numbers were measured beginning on Day 0,4 and 8 . On Day 0 , cell numbers were not different among treatments; however, by Day 4, cell number had decreased with GLE treatment as compared to the control. Day 8 showed the same results as Day 4 (Figure 3).

\section{GLE Enhanced AMPK Activity and Inhibited 3T3-L1 Adipogenesis}

Activity of AMPK was assessed by measuring the phosphorylated AMPK ( $\mathrm{p}-\mathrm{AMPK}$ ) level. Compared with the control, the GLE-treatment group exhibited significantly increased p-AMPK protein (Figure 4A). Moreover, after treatment with AMPKa siRNA and restoration of differentiation by GLE, the relative fatty accumulation of each group was determined. The results suggested that AMPKa siRNA can restore adipocyte differentiation with GLE treatment (Figure 4B).

\section{DISCUSSION}

In our previous studies, we showed that oral administration of guava leaf extract (GLE) had

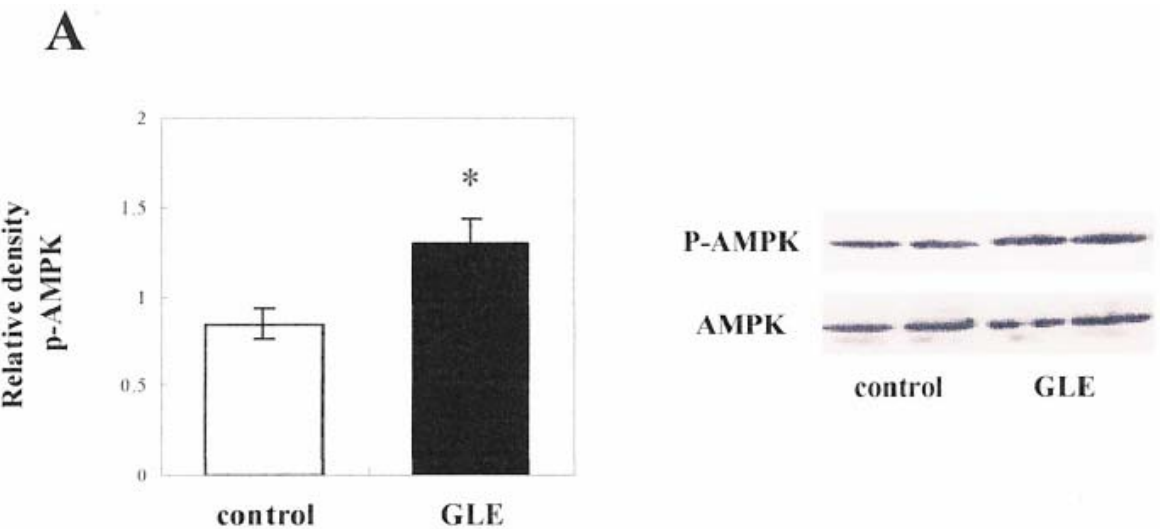

B

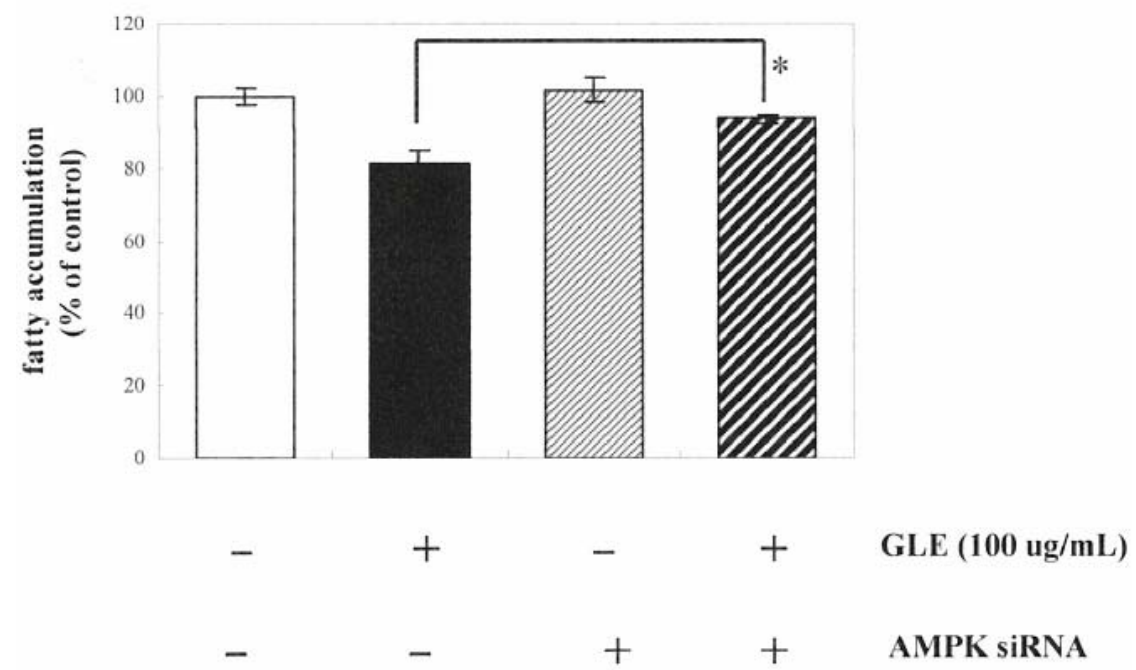

Figure 4: Effect of GLE on AMPK in 3T3-L1 cells.

After $24 \mathrm{hr}$ of GLE (100 ug/mL) treatment to confluence cells, cells were collected and lysed for Western blotting to determine the effect of GLE on AMPK activation (A). Phosphorylated AMPK was normalized to AMPK. After AMPK siRNA pretreatment and GLE-induced differentiation, the relative intracellular fatty accumulation of 3T3-L1 cells in each group was measured at Day 8 (B). Data are the means \pm SEM;. * $P<0.05$ vs control group. $n=3$ 
beneficial anti-obesity effects using SHRSP.ZLeprfa/lzmDmcr rats. We did not, however, clarify how to improve obesity by GLE administration. This study was designed to evaluate the mechanism of antiobesity by GLE using 3T3-L1 pre-adipocyte cell lines and we show that GLE inhibited 3T3-L1 adipocyte differentiation which is a key player in obesity and metabolic syndrome and may serve as a potential therapeutic target for treating obesity.

Pre-adipocytes exposed to multiple hormones and cytokines, including CAMP, insulin, and IGF-1, were able to fully mature into adipocytes [15]. Several transcription factors have been identified that act in a sequential fashion to promote adipocyte differentiation. PPARy and C/EBP families served as early markers of adipocyte differentiation. The C/EBP families include $\mathrm{C} / \mathrm{EBPa}, \mathrm{C} / \mathrm{EBP} \beta$ and C/EBPD. Transient expression of $\mathrm{C} / \mathrm{EBP} \beta$ and $\mathrm{C} / \mathrm{EBP} \delta$ occurs during the early stages of differentiation followed by induced expression of PPARy and C/EBPa. They then induce the expression of multiple adipocyte-specific genes and are important for terminal adipocyte differentiation [16]. Cells develop the fat accumulation ability at differentiation. Our studies demonstrated that GLE inhibits 3T3-L1 adipogenesis at a relatively early stage by regulating the expression of PPARy and C/EBPa mRNA. Moreover, GLE significantly suppressed aP2, which is a fatty acid binding protein downstream of PPARy, so we used late adipogenic markers.

On the other hand, previous studies have identified that mitotic clonal expansion was essential for 3T3-L1 differentiation. During the clonal expansion phase, cells are relieved of the growth arrest signal initiated upon cell confluence and undergo multiple rounds of DNA synthesis and mitosis, followed by exit from the cell cycle and the deduction of several adipose-specific genes. Tang et al. reported that $\mathrm{C} / \mathrm{EBP} \beta$ was required for clonal expansion [10]. In order to determine of effects on clonal expansion of GLE, we quantitated cell numbers and found that GLE had inhibitory effects on clonal expansion of 3T3-L1 cells.

Most interestingly, we identified that GLE treatment increased the expression of $\mathrm{p}$-AMPK, while AMPKa siRNA pretreatment increased adipocyte fatty accumulation. AMPK, an energy sensor, plays a key role in the regulation of fatty acid. Several researches have demonstrated that AMPK activation reduced body weight gain and decreased liver and plasma triglyceride in vivo [17]. Furthermore, in vitro, it was reported to affect AMPK in adipocyte differentiation.
Zhou et al. reported that A-769662, which is an AMPK activator, inhibited 3T3-L1 adipogenic differentiation by down-regulating the expression of C/EBP $\beta$ and $\mathrm{C} / \mathrm{EBP} \delta$, and hence inhibiting clonal expansion. Moreover, it blocked the expression of PPARy and $\mathrm{C} / \mathrm{EBPa}$ and downstream adipogenic genes. This compound also activated AMPK and hence phosphorylated and inactivated ACC, which is essential for lipid biosynthesis [18]. Susan et al. also demonstrated that an AMPK activator, 5aminoimidazole-4-carboxamide ribonucleoside (AICAR), inhibited the differentiation of 3T3-L1 adipocytes by blocking the expression of adipogenic markers and early clonal expansion [19]. The AMPK cascade is an important therapeutic target for obesity because it promotes the expression or phosphorylation of downstream proteins involved in energy metabolism. Some phytochemicals, such as epigallocatechin-3gallate [20], resveratrol [21], capsaicin and genistein [22], have demonstrated the activation of AMPK and inhibition of 3T3-L1 adipocyte lipogenesis. GLE may have a similar mechanism.

Taken together, these results suggest that GLE inhibited adipocyte differentiation by down-regulating the expression of PPARy and C/EBPa and downstream adipogenic genes, which are essential for adipocytes, and inhibited clonal expansion via AMPK activity; therefore, oral administration of GLE may lead to beneficial anti-obesity effect.

\section{COMPETING INTERESTS}

The authors declare that they have no competing interests.

\section{ABBREVIATIONS}

ACC = Acetyl-CoA carboxylase

AMPK = AMP-activated protein kinase

aP2 = adipocyte fatty acid binding protein

C/EBP- $\alpha=$ CCAAT/enhancer- binding protein $\alpha$

FFA $=$ free fatty acid

GLE = Guava leaf extract

IL-6 = interleukin-6

$\mathrm{PCR}=$ polymerase chain reaction

PPAR- $\gamma=$ peroxisome proliferators-activated receptor $\gamma$

TNF $=$ Tumor Necrosis Factor 


\section{REFERENCES}

[1] Gutierrez RM, Mitchell S, Solis RV. Psidium guajava: a review of its traditional uses, phytochemistry and pharmacology. J Ethnopharmacol 2008; 117: 1-27. http://dx.doi.org/10.1016/j.jep.2008.01.025

[2] Keji C. Understanding and treatment of diabetes mellitus by traditional Chinese medicine. Am J Chin Med 1981; 9: 93-94. http://dx.doi.org/10.1142/S0192415X81000123

[3] Begum S, Hassan SI, Ali SN, Siddiqui BS. Chemical constituents from the leaves of Psidium guajava. Nat Prod Res 2004; 18: 135-40. http://dx.doi.org/10.1080/14786410310001608019

[4] Begum S, Hassan SI, Siddiqui BS. Two new triterpenoids from the fresh leaves of Psidium guajava. Planta Med 2002; 68: $1149-52$. http://dx.doi.org/10.1055/s-2002-36353

[5] Lozoya X, Meckes M, Abou-Zaid M. Quercetin glycosides in Psidium guajava $L$. leaves and determination of a spasmolytic principle. Arch Med Res 1994; 25: 11-5.

[6] de Rezende FM, Furlan CM. Anthocyanins and tannins in ozone-fumigated guava trees. Chemosphere 2009; 76: 144550.

http://dx.doi.org/10.1016/j.chemosphere.2009.05.028

[7] Yoshitomi H, Guo X, Liu T,Gao M. Guava leaf extracts alleviate fatty liver via expression of adiponectin receptors in SHRSP.Z-Leprfa/lzm rats. Nutr Metab 9: 13. http://dx.doi.org/10.1186/1743-7075-9-13

[8] Farmer SR. Transcriptional control of adipocyte formation. Cell Metab 2006; 4: 263-73. http://dx.doi.org/10.1016/j.cmet.2006.07.001

[9] Rosen ED, Spiegelman BM. Molecular regulation of adipogenesis. Annu Rev Cell Dev Biol 2000; 16: 145-71. http://dx.doi.org/10.1146/annurev.cellbio.16.1.145

[10] Tang QQ, Otto TC, Lane MD. Mitotic clonal expansion: a synchronous process required for adipogenesis. Proc Natl Acad Sci USA 2003; 100: 44-9. http://dx.doi.org/10.1073/pnas.0137044100

[11] Towler MC, Hardie DG. AMP-activated protein kinase in metabolic control and insulin signaling. Circ Res 2007; 100: 328-41.

http://dx.doi.org/10.1161/01.RES.0000256090.42690.05

[12] Hardie DG. AMP-activated/SNF1 protein kinases: conserved guardians of cellular energy. Nat Rev Mol Cell Biol 2007; 8: 774-85.

http://dx.doi.org/10.1038/nrm2249

[13] Sim AT, Hardie DG. The low activity of acetyl-CoA carboxylase in basal and glucagon-stimulated hepatocytes is due to phosphorylation by the AMP-activated protein kinase and not cyclic AMP-dependent protein kinase. FEBS Lett 1988; 233: 294-8.

http://dx.doi.org/10.1016/0014-5793(88)80445-9

[14] Ruderman N, Prentki M. AMP kinase and malonyl-CoA: targets for therapy of the metabolic syndrome. Nat Rev Drug Discov 2004; 3: 340-51. http://dx.doi.org/10.1038/nrd1344

[15] MacDougald OA, Lane MD. Transcriptional regulation of gene expression during adipocyte differentiation. Annu Rev Biochem 1995; 64: 345-73. http://dx.doi.org/10.1146/annurev.bi.64.070195.002021

[16] Kudo M, Sugawara A, Uruno A, Takeuchi K, Ito $S$. Transcription suppression of peroxisome proliferatoractivated receptor gamma2 gene expression by tumor necrosis factor alpha via an inhibition of CCAAT/ enhancerbinding protein delta during the early stage of adipocyte differentiation. Endocrinology 2004; 145: 4948-56. http://dx.doi.org/10.1210/en.2004-0180

[17] Picard F, Kurtev M, Chung N, Topark-Ngarm A, Senawong T, Machado De Oliveira R, et al. Sirt1 promotes fat mobilization in white adipocytes by repressing PPAR-gamma. Nature 2004; 429: 771-76. http://dx.doi.org/10.1038/nature02583

[18] Zhou Y, Wang D, Zhu Q, Gao X, Yang S, Xu A, et al. Inhibitory effects of A-769662, a novel activator of AMPactivated protein kinase, on 3T3-L1 adipogenesis. Biol Pharm Bull 2009; 32: 993-98. http://dx.doi.org/10.1248/bpb.32.993

[19] Habinowski SA, Witters LA. The effects of AICAR on adipocyte differentiation of 3T3-L1 cells. Biochem Biophys Res Commun 2001; 286: 852-6. http://dx.doi.org/10.1006/bbrc.2001.5484

[20] Moon HS, Chung CS, Lee HG, Kim TG, Choi YJ, Cho CS Inhibitory effect of (-)-epigallocatechin-3-gallate on lipid accumulation of 3T3-L1 cells. Obesity (Silver Spring) 2007; 15: 2571-82. http://dx.doi.org/10.1038/oby.2007.309

[21] Chen S, Li Z, Li W, Shan Z, Zhu W. Resveratrol inhibits cell differentiation in 3T3-L1 adipocytes via activation of AMPK. Can J Physiol Pharmacol 89: 793-99.

[22] Hwang JT, Park IJ, Shin JI, Lee YK, Lee SK, Baik HW, et al. Genistein, EGCG, and capsaicin inhibit adipocyte differentiation process via activating AMP-activated protein kinase. Biochem Biophys Res Commun 2005; 338: 694-99. http://dx.doi.org/10.1016/j.bbrc.2005.09.195 